Psychotherapeut 2014 $\cdot 59: 273-274$

DOI 10.1007/s00278-014-1063-3

Online publiziert: 28. Juni 2014

(c) Springer-Verlag Berlin Heidelberg 2014

Georg Schomerus · Harald J. Freyberger

Klinik und Poliklinik für Psychiatrie und Psychotherapie, Universitätsmedizin

Greifswald am Helios Hanseklinikum Stralsund

\title{
Psychotherapie und Stigma
}

bessern kann (Rüsch et al. 2014; Schomerus 2010). Auch in der vorliegenden Ausgabe der Zeitschrift Psychotherapeut zeigt Rudolph, wie in der Psychotherapie mit dem Stigma von Übergewicht umgegangen werden kann. Andere Forschungsarbeiten in diesem Heft gehen dagegen über diese Fragen hinaus und spüren der Verbindung zwischen Psychotherapie und Stigma in einem größeren Kontext nach.

Prü $\beta$ et al. widmen sich der Frage, ob die Psychotherapeuten und Psychiater selbst Opfer von Stigmatisierung sind. Gibt es hier einen blinden Fleck der Stigmaforschung, die sich bisher v. a. um die von psychischer Krankheit Betroffenen gekümmert hat (Sibitz et al. 2013)? Insbesondere ärztliche Psychotherapeuten neigen dazu, wie wir aus vielen persönlichen Gesprächen wissen, sich von ihren medizinischen Kollegen als stigmatisiert zu erleben, obwohl ihre Akzeptanz vergleichsweise gut erscheint (Heuft et al.). Tatsächlich ist zunächst einmal das Wissen über die Psychotherapie in der Bevölkerung teilweise gering (Bluml et al. 2013), und mit der Person des Therapeuten werden zahlreiche Stereotype assoziiert. In einer systematischen Recherche der nationalen und internationalen Literatur bringen Prüß et al. Befunde zur möglichen Diskriminierung von Therapeuten in den $\mathrm{Zu}$ sammenhang etablierter Stigmatheorien. Deutlich wird dabei, dass allein aus dem Vorhandensein von Fehlinformationen und Stereotypen nicht automatisch auf eine Stigmatisierung geschlossen werden sollte. Stigmatisierung setzt faktische Benachteiligung sowie ein Machtgefälle zwischen einer Mehrheit und einer stigmatisierten Minderheit voraus. Fehlinformationen und Stereotype existieren dagegen über eine Vielzahl von Berufs- und Bevölkerungsgruppen und sollten nicht voreilig mit dem Begriff „Stigmatisierung“ skandalisiert werden. Initiativen zur „Entstigmatisierung" einer Berufsgruppe, wie sie beispielsweise von der World Psychiatric Association (WPA; Sartorius et al. 2010) verfolgt werden, wirken vor diesem Hintergrund beinahe eigennützig und scheinen den Blick auf die anhaltende und schwerwiegende Benachteiligung von Menschen mit psychischen Krankheiten im Gesundheitssystem und in der Gesellschaft zu verstellen.

Schäfer et al. widmen sich möglichen Behandlungsbarrieren bei einer besonders stigmatisierten Patientengruppe, den Konsumenten illegaler Drogen wie z. B. „Crystal“. Sie werten qualitative Ergebnisse einer Onlinebefragung einer großen Stichprobe von Methamphetaminkonsumenten aus. Dabei finden sie Hinweise auf wichtige Wechselbeziehungen zwischen psychiatrischen Komorbiditäten, Traumatisierungen und dem aktuellen Konsum. Deutlich wird ein hoher Behandlungsbedarf der Betroffenen, der allerdings gerade auf dem Gebiet der Psychotherapie aktuell in keiner Weise gedeckt werden kann. Die Autoren diskutieren deshalb die Frage, inwieweit Drogenkonsumenten im psychotherapeutischen Versorgungssystem diskriminiert werden. Inwieweit sind Psychotherapeuten aktiv an der Stigmatisierung einer Minderheit beteiligt?

In einem weiteren Beitrag dieses Themenheftes untersuchen Schlier u. Lincoln die Darstellung der Erkrankung Schizophrenie in deutschen Printmedien. Sie gehen dabei der Frage nach, inwieweit Berichte über das Krankheitsbild, aber auch der metaphorische Gebrauch des Worts chotherapie zumindest die persönlichen Fähigkeiten zum Umgang mit Stigma ver- 
Schizophrenie stigmatisierende Stereotype und Vorurteile transportieren. Diese Frage ist bei der Schizophrenie von besonderer Relevanz: Zum einen ist die Krankheitsbezeichnung ein ,iatrogenes“ Kunstwort, das von Eugen Bleuler 1911 erstmalig für die „Gruppe der Schizophrenien“ verwendet wurde (Bleuler 1911). Sollte dieses Wort diskriminierende Assoziierungen wecken, die nichts mit der Erkrankung, wohl aber etwas mit seiner altgriechischen Übersetzung („gespaltene Seele“) zu tun haben, wäre eine Umbenennung der Erkrankung notwendig. Auf der anderen Seite machen Schlier u. Lincoln auch auf negative Assoziationen aufmerksam, die nichts mit dem Begriff, sondern eher mit der medialen Überhöhung schwerer Krankheitszustände zu tun haben - etwa in der überproportionalen Darstellung von Gewalttaten. Schließlich stellen sie ihre Untersuchung in einen zeitlichen Kontext und vergleichen ihre Ergebnisse mit denen ähnlicher Untersuchungen aus den 1990er Jahren.

Eine weitere Arbeit des vorliegenden Hefts wendet sich wieder ganz den Psychotherapeuten $\mathrm{zu}$ - diesmal allerdings in der ungewohnten Rolle des Hilfeempfängers. Von Sydow (2014) untersucht mithilfe einer extensiven Literaturrecherche die Frage, inwieweit die psychotherapeutische Versorgung der Therapeuten ein Tabu darstellt, ob hier die Angst vor Stigmatisierung oder vor der Auseinandersetzung mit eigenen stigmatisierenden Haltungen die Inanspruchnahme von Hilfe verhindert. Diese Frage ist umso berechtigter, als berufliche Belastungen als Krankheitsursache in letzter Zeit - nicht zuletzt im Rahmen der Burn-out-Diskussion stärker in das Bewusstsein der Öffentlichkeit gelangt zu sein scheinen (Angermeyer et al. 2013c; Bahlmann et al. 2013. Insbesondere den Angehörigen therapeutischer Berufe werden dabei hohe psychische und somatische Belastungen attestiert (Grassel et al. 2013). Wie ist es nun um die Selbstfürsorge der Therapeuten bestellt? Und noch wichtiger: Könnten Therapeuten, die transparent auch mit eigener psychischer Krankheit umgehen, als Rollenmodelle zur Entstigmatisierung der Krankheiten beitragen? Hier nennt von Sydow Beispiele, die insgesamt Hoffnung machen. Vielleicht liegt hier eine wichtige
Möglichkeit, wie Psychotherapeuten über ihre Expertenrolle hinaus als Betroffene dem Stigma psychischer Krankheit etwas entgegensetzen können.

Stigma und Psychotherapie weisen also viele Berührungspunkte auf. Psychotherapeuten sind als Helfer bei der Bewältigung von Stigma gefragt, aber auch als Vorbilder beim Umgang mit eigenen psychischen Problemen. Vermutlich sollten die psychosomatische Medizin, Psychotherapie und die Psychiatrie nicht zu viele Ressourcen auf die Frage verwenden, ob sie selbst als Fach eine Benachteiligung erfahren, sondern sich weiter in den Dienst der nach wie vor schwer benachteiligten Betroffenen stellen. Inwieweit innerhalb der Psychotherapie Menschen mit schweren psychischen Krankheiten und insbesondere mit komorbiden Suchterkrankungen diskriminiert werden, ist eine drängende Frage, die vorerst offen bleibt.

\section{Korrespondenzadresse}

\section{PD Dr. Georg Schomerus}

Klinik und Poliklinik für Psychiatrie und Psychotherapie, Universitätsmedizin Greifswald am Helios Hanseklinikum Stralsund Rostocker Chaussee 70, 18437 Stralsund georg.schomerus@uni-greifswald.de

\section{Einhaltung ethischer Richtlinien}

Interessenkonflikt. Georg Schomerus und Harald J. Freyberger geben an, dass kein Interessenkonflikt besteht.

\section{Literatur}

Angermeyer MC, Matschinger H, Schomerus G (2013a) Attitudes towards psychiatric treatment and people with mental illness: changes over two decades. Br J Psychiatry 203:146-151

Angermeyer MC, Matschinger $\mathrm{H}$, Schomerus G (2013b) Public attitudes towards people with depression in times of uncertainty: results from three population surveys in Germany. Soc Psychiatry Psychiatr Epidemiol 48:1513-1518

Angermeyer MC, Schomerus G, Carta MG et al. (2013c) Burnout - ein deutsches Phänomen? Psychiatr Prax 40:425-429

Bahlmann J, Angermeyer MC, Schomerus G (2013) „Burnout" statt „Depression" - eine Strategie zur Vermeidung von Stigma? Psychiatr Prax 40:78-82

Bleuler E (1911) Dementia Praecox: oder Gruppe der Schizophrenien. Deuticke, Leipzig

BlumI V, Angermeyer MC, Holzinger A (2013) Assoziationen zur Psychoanalyse - Ergebnisse einer Repräsentativerhebung bei der Wiener Bevölkerung. Psychiatr Prax 40:130-134
Evans-Lacko S, Courtin E, Fiorillo A et al (2014) The state of the art in European research on reducing social exclusion and stigma related to mental health: a systematic mapping of the literature. Eur Psychiatry 2014 Apr 9. DOI 10.1016/j.eurpsy.2014.02.007

Grassel E, Lampen-Imkamp S, Lehrl S, Kahl KG (2013) Psychische und somatische Beschwerden bei Medizinstudierenden. Psychiatr Prax 40, 30-35

Rüsch N (2010) Reaktionen auf das Stigma psychischer Erkrankung. Z Psychiatr Psychol Psychother 58:287-297

Rüsch N, Abbruzzese E, Hagedorn E et al (2014) Efficacy of coming out proud to reduce stigma's impact among people with mental illness: pilot randomised controlled trial. Br J Psychiatry, DOI 10.1192/ bjp.bp.113.135772

Sartorius N, Gaebel W, Cleveland HR et al (2010) WPA guidance on how to combat stigmatization of psychiatry and psychiatrists. World Psychiatry 9:131144

Schomerus G (2010) The stigma of mental illness - a topic for psychotherapy? Z Psychiatr Psychol Psychother 58:253-255

Schomerus G, Angermeyer MC (2013) Psychiatrie endlich entstigmatisiert? Psychiatr Prax 40:59-61

Schomerus G, Auer C, Rhode D et al (2012) Personal stigma, problem appraisal and perceived need for professional help in currently untreated depressed persons. J Affect Disord 139:94-97

Schomerus G, Matschinger H, Angermeyer MC (2015) Attitudes towards alcohol dependence and affected persons - the persistence of negative stereotypes and illness beliefs 1990 - 2011. Eur Addiction Res (in press)

Sibitz I, Friedrich ME, Unger A et al (2013) Internalisiertes Stigma bei Schizophrenie: Validierung der deutschen Version der Internalized Stigma of Mental Illness-Skala (ISMI). Psychiatr Prax 40:83-91 